\title{
Lipid Emulsion Therapy in Lipophilic or Hydrophilic Drug Intoxication: The Last Weapon in Our Arsenal
}

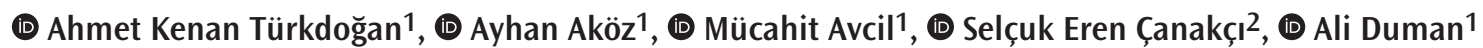 \\ 1Department of Emergency Medicine, Adnan Menderes University Faculty of Medicine, Aydın, Turkey \\ ${ }^{2}$ Clinic of Emergency, Kütahya Doç. Dr. Mustafa Kalemli Tavșanlı State Hospital, Kütahya, Turkey
}

\begin{abstract}
Aim: Previous case reports have described the administration of lipid emulsion therapy in lipophilic drug intoxication cases. In this study, we wanted to contribute to the literature that lipid emulsion therapy could also be given lipid as the last weapon in not only lipophilic drug intoxication but also all intoxication cases with worsening general condition.

Materials and Methods: A total of 65 patients, who presented to the emergency room and received lipid therapy between January 1, 2014 and January 1, 2017, were included in this study. Each patient was given a $20 \%$ ClinOleic (Baxter) infusion of $1.5 \mathrm{~mL} / \mathrm{kg}$ for $1-3 \mathrm{minutes}$ and then $100 \mathrm{~mL} / \mathrm{h}(0.025 \mathrm{~mL} / \mathrm{kg} / \mathrm{min})$. Toxic drugs were divided into low or high permeability groups according to their lipid/water partition coefficients (LogP).

Results: Of the 65 patients, $55.4 \%(n=36)$ were female and $44.6 \%(n=29)$ were male. These patients were grouped according to a lipid/water cut-off value of 1.72. The lipid therapy was administered in addition to antidotal therapy in two patients in the hydrophilic group and in five patients in the lipophilic group. The only variable that was significantly restored 12 hours after the lipid therapy was the respiratory rate, which was 16.0 (range, 15.5-17.3)/min in the hydrophilic group and 20.0 (range, 18.0-22.0)/min in the lipophilic group ( $p=0.003$ ).

Conclusion: We believe that lipid therapy can be used as a last resort in intoxication cases, especially in patients with low Glasgow coma scale scores and worsened vital signs despite antidotal and extracorporeal therapies, regardless of whether the causative agent is hydrophilic or lipophilic.
\end{abstract}

Keywords: Lipophilicity, lipid emulsion therapy, toxicity, hydrophilicity, lipid/water partition coefficient, emergency service

\section{Introduction}

For the first time in 1998, the infusion of soybean oil used in total parenteral nutrition solutions showed that it could prevent cardiovascular collapse resulting from an overdose of bupivacaine in anesthetized rats and could improve resuscitation (1). In another experimental model, spontaneous circulation was restored in all lipid-treated animals following a bupivacaine challenge, but it was not restored in any of the saline-treated control subjects (2). The first case report describing the use of intravenous lipid emulsion (ILE) therapy as a rescue or antidotal therapy for acute intoxication was published in 2006 (3). In recent years, it has become one of the most recommended treatment methods for patients who have experienced cardiac arrest or those with high hemodynamic risk following xenobiotic toxicity (4).

The mechanism of action of ILE in the management of intoxication is not yet fully understood; however, numerous mechanisms are thought to contribute to its activity. The emulsion acts as a lipid sink that surrounds and neutralizes a lipophilic drug molecule. Several in vitro and in vivo studies have confirmed the benefits of the lipid sink impact (5-7). The in vitro models have suggested 
that the mechanism underlying ILE binding depends on the lipid/water partition coefficient and distribution volume. The lipid/water coefficient defines how easily a drug moves between the water and lipid medium (8). Drugs with a LogP $>1.72$ are classified as having high permeability and those with a LogP $<1.72$ are considered to have low permeability $(9,10)$.

In this retrospective study, we aimed to share the demographic data and outcomes of patients treated with ILE therapy based on lipophilicity in the emergency department in the light of the literature. We were especially interested in patients with poor general status despite ongoing initial antidotal and extracorporeal therapies, regardless of the lipophilicity of the toxic agent.

\section{Materials and Methods}

\section{Trial Design and Participants}

A total of 73 patients, who were admitted to the emergency room and received ILE therapy between January 1, 2014 and January 1,2017 , were retrospectively evaluated with the approval of the local ethics committee (53043469-050.04.04/2017-1071). A total of 65 patients with accessible data were included in this research.

According to the dosing scheme recommended by the American College of Medical Toxicology, each patient was administered a bolus of $20 \%$ ClinOleic (Baxter) at $1.5 \mathrm{~mL} / \mathrm{kg}$ for $1-3$ minutes (11). The infusion continued at $100 \mathrm{~mL} / \mathrm{h}(0.025 \mathrm{~mL} / \mathrm{kg} / \mathrm{min})$ until the hemodynamic variables returned to normal. The lipid/water partition coefficients (LogP) of the drugs taken at toxic doses were determined and they were divided into hydrophilic and lipophilic groups $(9,10)$.

\section{Follow-up}

All patients were followed-up in the emergency intensive care and emergency observation units. Demographics, admission data, intensive care follow-up, extracorporeal treatments and outcomes were evaluated. Since the patients receiving ILE therapy in our service were followed-up for one month, any additional complaints were evaluated for one month after discharge.

\section{Statistical Analysis}

The normality of continuous variables was evaluated by Kolmogorov-Smirnov test. The descriptive statistics of the normally distributed variables were expressed as mean \pm standard deviation, and an Independent Samples t-test was used for comparisons between the groups. The descriptive statistics of the non-normally distributed variables were expressed as median (25\%-75\%), and Mann-Whitney U test was used for comparisons between the groups. P value $<0.05$ was considered statistically significant.

\section{Results}

Among the 73 patients who underwent ILE therapy due to intoxication, the data of 65 patients was obtained. Of these patients, 55.4\% $(n=36)$ were females and $44.6 \%(n=29)$ were males. The mean age was $32.5 \pm 12.6$ years for females and $35.0 \pm 14.7$ years for males.

The cut-off value for lipid/water partition coefficient was 1.72, and the patients were divided into hydrophilic and lipophilic groups accordingly (Table 1). Of the 59 patients in the lipophilic

\section{Table 1. Intoxication agents and their LogP values}

\begin{tabular}{|c|c|c|}
\hline Lipophilicity & Toxic agent & LogP \\
\hline \multirow[t]{25}{*}{$\begin{array}{l}\text { Lipophilic, LogP } \\
\geq 1.72(1,2)\end{array}$} & Tetrahydrocannabinol & 7.22 \\
\hline & Amitriptyline & 4.81 \\
\hline & Imipramine & 4.80 \\
\hline & Biperiden & 4.28 \\
\hline & Fluoxetine & 4.05 \\
\hline & Paroxetine & 3.70 \\
\hline & Dexibuprofen & 3.67 \\
\hline & Opipramole & 3.45 \\
\hline & Clozapine & 3.23 \\
\hline & Bupropion & 3.12 \\
\hline & Escitalopram & 3.08 \\
\hline & Phenytoin & 2.47 \\
\hline & Olanzapine & 3.00 \\
\hline & Valproic Acid & 2.75 \\
\hline & Warfarin & 2.70 \\
\hline & Mirtazapine & 2.70 \\
\hline & Alprazolam & 2.65 \\
\hline & Carbamazepine & 2.45 \\
\hline & Venlafaxine & 2.38 \\
\hline & Malathion & 2.36 \\
\hline & Amlodipine & 2.22 \\
\hline & Quetiapine & 2.09 \\
\hline & Metoprolol & 1.87 \\
\hline & Bisoprolol & 1.87 \\
\hline & Amphetamine & 1.85 \\
\hline \multicolumn{3}{|c|}{ Hydrophilic, LogP <1.72 $(1,2)$} \\
\hline & Paracetamol & 0.46 \\
\hline & Methylphenidate & 0.20 \\
\hline & Theophylline & -0.13 \\
\hline & Monocrotophos & -0.40 \\
\hline & Pregabalin & -0.55 \\
\hline
\end{tabular}


Table 2. Distributions of length of hospitalization, Glasgow coma scale scores, mean arterial pressures and respiratory rates according to toxic agent group

\begin{tabular}{|c|c|c|c|}
\hline & Lipophilic & Hydrophilic & $p$ \\
\hline \multicolumn{4}{|l|}{ Length of hospitalization } \\
\hline Emergency intensive care & $2.0(1.0-4.0)(n=59)$ & $2.5(0.8-6.0)(n=6)$ & 0.670 \\
\hline Observation unit & $1.0(1.0-2.0)(n=59)$ & $1.5(1.0-2.5)(n=6)$ & 0.479 \\
\hline Total (day) & $3.0(2.0-5.0)(n=59)$ & $3.5(2.8-7.0)(n=6)$ & 0.365 \\
\hline \multicolumn{4}{|l|}{ GCS } \\
\hline Admission & $13.0(4.5-15.0)(n=48)$ & $15.0(8.5-15.0)(n=5)$ & 0.213 \\
\hline Post-treatment $12^{\text {th }}$ hour & $15.0(14.0-15.0)(n=48)$ & $15.0(11.0-15.0)(n=5)$ & 0.867 \\
\hline Post-treatment $24^{\text {th }}$ hour & $15.0(14.0-15.0)(n=48)$ & $15.0(14.0-15.0)(n=5)$ & 0.955 \\
\hline \multicolumn{4}{|l|}{ Mean arterial pressure } \\
\hline Admission & $88.9 \pm 16.3(n=45)$ & $95.7 \pm 18.0(n=5)$ & 0.388 \\
\hline Post-treatment $12^{\text {th }}$ hour & $83.3 \pm 14.2(n=45)$ & $83.3 \pm 10.8(n=5)$ & 0.997 \\
\hline Post-treatment $24^{\text {th }}$ hour & $83.4 \pm 15.1(n=45)$ & $90.7 \pm 10.6(n=5)$ & 0.302 \\
\hline \multicolumn{4}{|l|}{ Respiratory rate } \\
\hline Admission & $20.0(18.0-22.0)(n=51)$ & $16.0(15.5-17.3)(n=6)$ & 0.003 \\
\hline Post-treatment $12^{\text {th }}$ hour & $18.0(16.0-21.0)(n=51)$ & $21.0(15.5-25.5)(n=6)$ & 0.255 \\
\hline Post-treatment $24^{\text {th }}$ hour & $19.0(15.5-22.0)(n=51)$ & $18.0(14.0-20.0)(n=6)$ & 0.417 \\
\hline
\end{tabular}

group, 56 presented with intake of only lipophilic drugs and three presented with intake of both hydrophilic and toxic lipophilic drugs. Of the six patients in the lipophilic group, two presented with intake of only hydrophilic drugs and four presented with intake of both lipophilic and toxic hydrophilic drugs.

The length of hospitalization, Glasgow coma scale (GCS) score, mean arterial pressure (MAP) and respiratory rate of the patients are shown in Table 2 . The respiratory rate was significantly higher in the lipophilic group than in the hydrophilic group $(p=0.003)$. Thirty-six patients were in the mono-drug group and 29 were in the poly-drug group. Three patients died during treatment and one patient developed pancreatitis. Mortality occurred due to insecticides in two patients and quetiapine in one patient. When the patients were classified according to the administered extracorporeal therapy, plasmapheresis was administered in one patient in the hydrophilic group and in two in the lipophilic group. Hemoperfusion was administered in four patients, hemofiltration in one patient, and hemodialysis in one patient in the lipophilic group.

ILE therapy was administered in addition to antidotal therapy in two patients in the hydrophilic group and five patients in the lipophilic group. The reasons for the ILE administration in addition to the antidotal therapy were low GCS score in three patients, the development of tachypnea in two patients and hypotension in two patients.

The vital signs and examination results of the patients at the time of admission were compared between the lipophilic and hydrophilic groups. The only statistically significant finding was the respiratory rate, which was 16.0 (range, 15.5-17.3)/min in the hydrophilic group and 20.0 (range, 18.0-22.0)/min in the lipophilic group $(p=0.003)$. The only variable that was restored after the neurological and vital sign examinations 12 hours after admission was the respiratory rate.

\section{Discussion}

One of the results making this study unique was the fact that ILE therapy could be applied regardless of whether the patient was overdosed with a lipophilic or hydrophilic substance. The other reason was that the respiratory rate could be used to evaluate the response of a patient to ILE administration. The only variable restored was the respiratory rate, which improved at 12 hours in the lipophilic group and 24 hours in the hydrophilic group. The delayed elevation of the respiratory rate in the hydrophilic group could be explained by late passage of drugs into the central nervous system. However, the decrease in the respiratory rate 24 hours after the ILE therapy could be attributed to the indication of lipid activity in the hydrophilic group.

One theory suggested that local anesthetics suspended the transport of fatty acids to cardiac mitochondria, thus decreasing the energy supply. Fatty acids provide a readily available energy resource for the myocardium, thus improving the function of the heart (12). However, this theory is insufficient to explain the effects of ILE therapy that we encountered in the non-local anesthetic and $\log P<1.72$ drug intoxication patients. We believe that this effect is associated with all drugs according to the LogP value of the toxic agent. 
In their study, Levine et al. (13) reported a case of acute pancreatitis and acute respiratory distress in a 13-year-old female patient who was given a lipid emulsion dose recommended by the American Society of Regional Anesthesia and Pain Medicine following tricyclic antidepressant overdose. The lipase level peaked at $1.849 \mathrm{U} / \mathrm{L}$ on the $5^{\text {th }}$ day of lipid administration. In our series of 65 patients, one patient among the patients who received IV organophosphate as a complication had a mild amylase increase. In addition, allergy developed in one patient who had escitalopram overdose.

In their review, Cao et al. (14) classified the drugs taken according to the $\log P=2$ classification. According to this study, metoprolol remained in the hydrophilic group, even though it was described as moderately lipophilic in the literature (15-18). In our study metoprolol remained in the lipophilic part of the classification.

No significant difference was found between the groups in terms of length of hospitalization, GCS and MAP values at 12 and 24 hours. However, there was an increase in the GCS score 12 hours after ILE administration compared to the admission scores in both groups. Although some of the case reports stated that ILE therapy was administered at lower GCS scores, the overall evaluation of the GCS scores was another important part of our study.

Poly-drug intake is unknown in the literature. In our study, $44.6 \%$ $(n=29)$ of the patients given ILE therapy presented with poly-drug intake. Four of the six patients in the hydrophilic group were admitted with lipophilic drug intake in addition to the intake of toxic hydrophilic drugs. This indicates that patients can receive additional lipophilic drugs despite the fact that the causative agent was hydrophilic, and thus, the toxic picture might worsen.

Three of the patients died during the treatment, and of those, the mortality occurred due to insecticide intake in two patients and quetiapine intake in one patient. It has been reported in the literature that low-dose lipid therapy could be useful in case of quetiapine and insecticide intoxications $(19,20)$. In our study, the reasons for the response failure of ILE therapy could be the low GCS scores at admission $(3,4,3)$ and late presentation to emergency service.

In one study, combined use of ILE therapy and extracorporeal treatment methods did not provide additional benefits in clinical practice (21). The patients were classified based on the administered extracorporeal therapy: plasmapheresis was administered in three patients, hemoperfusion in four patients, hemofiltration in one patient, and hemodialysis in one patient. Lipid therapy was applied in patients in whom the extracorporeal therapy would be delayed.

Filter occlusion was not observed in any of the patients because ILE was discontinued one hour before the extracorporeal therapy. The ILE therapy was administered in addition to the antidotal treatment in seven patients due to low GCS scores in three patients, the development of tachypnea in two patients, and hypotension in two patients.

\section{Study Limitations}

This study had several limitations. For example, it was designed as a retrospective study without pediatric patients. In addition, time from drug intake to hospital admission and time from admission to decontamination were not determined.

\section{Conclusion}

In conclusion, we believe that ILE therapy can be used as a last resort treatment regimen in cases of delayed antidotal and extracorporeal treatments, regardless of the lipophilicity of the toxic agent, especially in patients with a poor general status. In addition, the respiratory rate can be used to assess the response of a patient to ILE therapy. Further large-scale studies are needed both for the demonstration of the action mechanism and implementation in practice.

\section{Ethics}

Ethics Committee Approval: A total of 73 patients, who were admitted to the emergency room and received ILE therapy between January 1, 2014 and January 1, 2017, were retrospectively evaluated with the approval of the local ethics committee (53043469-050.04.04/2017-1071).

Informed Consent: Retrospective study.

Peer-review: Externally and internally peer-reviewed.

\section{Authorship Contributions}

Concept:A.K.T., A.A., M.A., S.E.C.., AD., Design:A.K.T., A.A., M.A., S.E.Ç.,A.D., Data Collection or Processing: A.K.T., A.A., M.A., S.E.C.., A.D., Analysis or Interpretation: A.K.T., A.A., M.A., S.E.C,., A.D., Literature Search: A.K.T., A.A., M.A., S.E.C., A.D., Writing: A.K.T., A.A., M.A., S.E.Ç., A.D.

Conflict of Interest: No conflict of interest was declared by the authors.

Financial Disclosure: The authors declared that this study received no financial support.

\section{References}

1. Weinberg GL, VadeBoncouer T, Ramaraju GA, Garcia-Amaro MF, Cwik MJ. Pretreatment or resuscitation with a lipid infusion shifts the dose-response to bupivacaine-induced asystole in rats. Anesthesiology. 1998;88:1071-5.

2. Weinberg G, Ripper R, Feinstein DL, Hoffman W. Lipid emulsion infusion rescues dogs from bupivacaine-induced cardiac toxicity. Reg Anesth Pain Med. 2003;28:198-202.

3. Jamaty C, Bailey B, Larocque A, Notebaert E, Sanogo K, Chauny JM. Lipid emulsions in the treatment of acute poisoning: a systematic review of human and animal studies. Clin Toxicol (Phila). 2010;48:1-27. 
4. Christian MR, Pallasch EM, Wahl M, Mycyk MB. Lipid rescue 911: Are poison centers recommending intravenous fat emulsion therapy for severe poisoning? J Med Toxicol. 2013;9:231-4.

5. Weinberg G, Lin B, Zheng S, Di Gregorio G, Hiller D, Ripper R, et al. Partitioning effect in lipid resuscitation: further evidence for the lipid sink. Crit Care Med. 2010;38:2268-9.

6. Mazoit JX, Le Guen R, Beloeil H, Benhamou D. Binding of longlasting local anesthetics to lipid emulsions. Anesthesiology. 2009;110:380-6.

7. Samuels TL, Willers JW, Uncles DR, Monteiro R, Halloran C, Dai H. In vitro suppression of drug-induced methaemoglobin formation by Intralipid $\left({ }^{\circledR}\right)$ in whole human blood: observations relevant to the 'lipid sink theory'. Anaesthesia. 2012;67:23-32.

8. French D, Smollin C, Ruan W, Wong A, Drasner K, Wu AH. Partition constant and volume of distribution as predictors of clinical efficacy of lipid rescue for toxicological emergencies. Clin Toxicol (Phila). 2011;49:801-9.

9. Kasim NA, Whitehouse M, Ramachandran C, Bermejo M, Lennernäs $\mathrm{H}$, Hussain AS, et al. Molecular properties of WHO essential drugs and provisional biopharmaceutical classification. Mol Pharm. 2004;1:85-96.

10. Takagi T, Ramachandran C, Bermejo M, Yamashita S, Yu LX, Amidon GL. A provisional biopharmaceutical classification of the top 200 oral drug products in the United States, Great Britain, Spain, and Japan. Mol Pharm. 2006;3:631-43.

11. American College of Medical Toxicology. ACMT Position Statement: Interim Guidance for the Use of Lipid Resuscitation Therapy. J Med Toxicol. 2011;7:81-2.
12. Gueret G, Pennec JP, Arvieux CC. Hemodynamic effects of intralipid after verapamil intoxication may be due to a direct effect of fatty acids on myocardial calcium channels. Acad Emerg Med. 2007;14:761.

13. Levine M, Brooks DE, Franken A, Graham R. Delayed-onset seizure and cardiac arrest after amitriptyline overdose, treated with intravenous lipid emulsion therapy. Pediatrics. 2012;130:432-8.

14. Cao D, Heard K, Foran M, Koyfman A. Intravenous lipid emulsion in the emergency department:a systematic review of recent literature. J Emerg Med. 2015;48:387-97.

15. Westerlund A. Central nervous system side-effects with hydrophilic and lipophilic beta-blockers. Eur J Clin Pharmacol. 1985;28(Suppl):73-6.

16. Ahmed Al, van Mierlo PJ, van Waarde JA, Jansen PA. Hallucinations and vivid dreams by use of metoprolol. Tijdschr Psychiatr. 2010;52:117-21.

17. Cove-Smith JR, Kirk CA. CNS-related side-effects with metoprolol and atenolol. Eur J Clin Pharmaco. 1985;28(Suppl):69-72.

18. Sirois FJ. Visual hallucinations and metoprolol. Psychosomatics. 2006;47:5378.

19. Purg D, Markota A, Grenc D, Sinkovic A. Low-dose intravenous lipid emulsion for the treatment of severe quetiapine and citalopram poisoning. Arh Hig Rada Toksikol. 2016;67:164-6.

20. Turkdogan KA, Duman A, Avcil M, Akoz A, Canakcı SE, Kantekin B. Treatment methods following suicidal self-administration of IV organophosphate: What can additional lipid administration change? Am J Emerg Med. 2017;35:1388.

21. Karaman K, Türkdoğan KA, Deniz AT, CanakcI SE. Which is the best in carbamazepine overdose? Clin Case Rep. 2017;5:1612-5. 